\title{
The Hawking temperature in the context of dark energy for Reissner-Nordstrom and Kerr background
}

\author{
Goutam Manna ${ }^{1, \mathrm{a}}$, Debashis Gangopadhyay ${ }^{2, \mathrm{~b}}$ \\ ${ }^{1}$ Department of Physics, Prabhat Kumar College, Contai, Purba Medinipur 721401, West Bengal, India \\ ${ }^{2}$ Department of Physics, Ramakrishna Mission Vivekananda University, P.O.-Belur Math, Howrah 711202, West Bengal, India
}

Received: 19 January 2014 / Accepted: 5 March 2014 / Published online: 18 March 2014

(C) The Author(s) 2014. This article is published with open access at Springerlink.com

\begin{abstract}
For emergent gravity metrics, the presence of dark energy modifies the Hawking temperature. We show that for the spherically symmetric Reissner-Nordstrom background metric, the emergent metric can be mapped into a Robinson-Trautman black hole. Allowed values of the dark energy density follow from rather general conditions. For some allowed values of the dark energy density this black hole can have zero Hawking temperature, i.e. the black hole does not radiate. For a Kerr background along $\theta=0$, the emergent black hole metric satisfies Einstein's equations for large $r$ and always radiates. Our analysis is done in the context of emergent gravity metrics having $k$-essence scalar fields $\phi$ with a Born-Infeld type lagrangian. In both cases the scalar field $\phi(r, t)=\phi_{1}(r)+\phi_{2}(t)$ also satisfies the emergent gravity equations of motion for $r \rightarrow \infty$ and $\theta=0$.
\end{abstract}

\section{Introduction}

In [1] it has been shown that the Hawking temperature [214] is modified in the presence of dark energy. In [1] this was shown for an emergent gravity metric $\tilde{G}_{\mu \nu}$ having $k$-essence scalar fields $\phi$ with a Born-Infeld type lagrangian and with the gravitational metric as Schwarzschild. The lagrangian for $k$-essence scalar fields contain non-canonical kinetic terms. The general form for such lagrangians is proportional to $F(X)$ with $X=\frac{1}{2} g^{\mu \nu} \nabla_{\mu} \phi \nabla_{\nu} \phi$. Relevant literature for such fields in cosmology, inflation, dark matter, dark energy, and strings can be found in [15-35].

The motivation of this work is to calculate the Hawking temperature for an emergent gravity metric in the presence of dark energy and which is also a black hole metric. We consider two cases, i.e., when the gravitational metric is a (a) Reissner-Nordstrom black hole metric and (b) Kerr black hole metric. As explained in detail in Sect. 2, $\tilde{G}_{\mu \nu}$ contains

\footnotetext{
a e-mail: goutammanna.pkc@gmail.com

b e-mail: debashis@rkmvu.ac.in
}

the dark energy field $\phi$ and this should satisfy the emergent gravity equations of motion. Again, for $\tilde{G}_{\mu \nu}$ to be a black hole metric, it has to satisfy the Einstein field equations. In Ref. [1] this was shown by mapping the emergent gravity metric (having Schwarzschild background) into a BarriolaVilenkin black hole metric which again satisfies the Einstein equations. Here we find that for the case of an RN background the emergent gravity metric can be exactly mapped onto a Robinson-Trautman black hole so that the Einstein equations are automatically satisfied. However, the $k$-essence matter fields satisfy the emergent gravity equations of motion only for $\theta=0$. For the Kerr case, the emergent metric satisfies Einstein equations for large $r$, while the dark energy field $\phi$ satisfies the emergent gravity equations of motion again only for $\theta=0$.

In this context we clarify that the Hawking temperature is spherically symmetric from very general conditions and taking $\theta=0$ does not therefore affect this property of the Hawking temperature. We will elaborate on this in a more quantitative way in Sects. 3 and 5. The formalism for emergent gravity used is as described in [36-39].

\section{Emergent gravity}

The emergent gravity minimal action for background metric $g_{\mu \nu}$ is

$S_{k}\left[\phi, g_{\mu \nu}\right]=\int \mathrm{d}^{4} x \sqrt{-} g L(X, \phi)$.

The energy momentum tensor is

$T_{\mu \nu} \equiv \frac{2}{\sqrt{-g}} \frac{\delta S_{k}}{\delta g^{\mu \nu}}=L_{X} \nabla_{\mu} \phi \nabla_{\nu} \phi-g_{\mu \nu} L$,

$L_{\mathrm{X}}=\frac{\mathrm{d} L}{\mathrm{~d} X}, \quad L_{\mathrm{XX}}=\frac{\mathrm{d}^{2} L}{\mathrm{~d} X^{2}}, \quad L_{\phi}=\frac{\mathrm{d} L}{\mathrm{~d} \phi}$ and $\nabla_{\mu}$ is the covariant derivative with respect to the metric $g_{\mu \nu}$. The equation of motion is 


$$
-\frac{1}{\sqrt{-g}} \frac{\delta S_{k}}{\delta \phi}=\tilde{G}^{\mu \nu} \nabla_{\mu} \nabla_{\nu} \phi+2 X L_{X \phi}-L_{\phi}=0,
$$

where

$\tilde{G}^{\mu \nu} \equiv L_{X} g^{\mu \nu}+L_{X X} \nabla^{\mu} \phi \nabla^{\nu} \phi$

and $1+\frac{2 X L_{X X}}{L_{X}}>0$.

Carrying out the conformal transformation $G^{\mu \nu} \equiv \frac{c_{s}}{L_{x}^{2}} \tilde{G}^{\mu \nu}$, with $c_{s}^{2}(X, \phi) \equiv\left(1+2 X \frac{L_{X X}}{L_{X}}\right)^{-1} \equiv$ sound speed, the inverse metric of $G^{\mu \nu}$ is obtained as

$G_{\mu \nu}=\frac{L_{X}}{c_{s}}\left[g_{\mu \nu}-c_{s}^{2} \frac{L_{X X}}{L_{X}} \nabla_{\mu} \phi \nabla_{\nu} \phi\right]$.

Another conformal transformation, $\bar{G}_{\mu \nu} \equiv \frac{c_{s}}{L_{X}} G_{\mu \nu}$, gives

$\bar{G}_{\mu \nu}=g_{\mu \nu}-\frac{L_{X X}}{L_{X}+2 X L_{X X}} \nabla_{\mu} \phi \nabla_{\nu} \phi ;$

$L_{X} \neq 0$ for the sound speed $c_{s}^{2}$ to be positive definite and only then (1)-(4) will be physically meaningful. This works as follows. $L_{X}=0$ means that $L$ is independent of $X$ so that in (1), $L(X, \phi) \equiv L(\phi)$. Then $L$ becomes pure potential and the very definition of $k$-essence fields becomes meaningless because such fields correspond to lagrangians where kinetic energy dominates over potential energy. Also the very concept of minimal coupling of $\phi$ to $g_{\mu \nu}$ becomes redundant and (1) gets meaningless; (4)-(6) become ambiguous.

For the non-trivial configurations of $\phi, \partial_{\mu} \phi \neq 0$ and $\bar{G}_{\mu \nu}$ are not conformally equivalent to $g_{\mu \nu}$. Therefore, this $\phi$ field has properties different from the canonical scalar fields defined with $g_{\mu \nu}$, and the local causal structure is also different from those defined with $g_{\mu \nu}$. Again, if $L$ is not an explicit function of $\phi$, then the equation of motion (3) is replaced by

$-\frac{1}{\sqrt{-g}} \frac{\delta S_{k}}{\delta \phi}=\bar{G}^{\mu \nu} \nabla_{\mu} \nabla_{\nu} \phi=0$.

We take the lagrangian as $L=L(X)=1-V \sqrt{1-2 X}$. This is a particular case of the BI lagrangian, $L(X, \phi)=$ $1-V(\phi) \sqrt{1-2 X}$, for $V(\phi)=V=$ constant and $V<<$ kinetic energy of $\phi$ i.e. $V<<(\dot{\phi})^{2}$. This is typical for the $k$-essence field where the kinetic energy dominates over the potential energy. Then $c_{s}^{2}(X, \phi)=1-2 X$. For scalar fields $\nabla_{\mu} \phi=\partial_{\mu} \phi$. Thus (6) becomes

$\bar{G}_{\mu \nu}=g_{\mu \nu}-\partial_{\mu} \phi \partial_{\nu} \phi$.

Note the rationale of using two conformal transformations: the first is used to identify the inverse metric $G_{\mu \nu}$, while the second realizes the mapping onto the metric given in (8) for the lagrangian $L(X)=1-V \sqrt{1-2 X}$.

\section{The Reissner-Nordstrom case and mapping onto the Robinson-Trautman type metric}

First consider the gravitational metric $g_{\mu \nu}$ to be ReissnerNordstrom and denote $\partial_{0} \phi \equiv \dot{\phi}, \partial_{r} \phi \equiv \phi^{\prime}$. Assuming that the $k$-essence field $\phi(r, t)$ is spherically symmetric one has

$$
\begin{aligned}
\bar{G}_{00} & =g_{00}-\left(\partial_{0} \phi\right)^{2}=1-2 G M / r+Q^{2} / r^{2}-\dot{\phi}^{2}, \\
\bar{G}_{11} & =g_{11}-\left(\partial_{r} \phi\right)^{2}=-\left(1-2 G M / r+Q^{2} / r^{2}\right)^{-1}-\left(\phi^{\prime}\right)^{2}, \\
\bar{G}_{22} & =g_{22}=-r^{2} \\
\bar{G}_{33} & =g_{33}=-r^{2} \sin ^{2} \theta \\
\bar{G}_{01} & =\bar{G}_{10}=-\dot{\phi} \phi^{\prime} .
\end{aligned}
$$

For the R-N metric, $g_{00}=\left(1-2 G M / r+Q^{2} / r^{2}\right) ; g_{11}=$ $-\left(1-2 G M / r+Q^{2} / r^{2}\right)^{-1} ; g_{22}=-r^{2} ; g_{33}=-r^{2} \sin ^{2} \theta$; $g_{i j}(i \neq j)=0$.

Note that the RN metric is spherically symmetric. The emergent gravity metric (9) contains additional terms but all these are independent of $\theta$. So the emergent metric is also spherically symmetric. Thus, we might as well consider $\theta=0$. Then the emergent gravity line element becomes

$$
\begin{aligned}
\mathrm{d} s_{\mathrm{RN}, \theta=0}^{2}= & \left(1-2 G M / r+Q^{2} / r^{2}-\dot{\phi}^{2}\right) \mathrm{d} t^{2} \\
& -\left(\left(1-2 G M / r+Q^{2} / r^{2}\right)^{-1}+\left(\phi^{\prime}\right)^{2}\right) \mathrm{d} r^{2} \\
& -2 \dot{\phi} \phi^{\prime} \mathrm{d} t \mathrm{~d} r .
\end{aligned}
$$

Making a coordinate transformation from $(t, r)$ to $(\omega, r)$ along $\theta=0$ such that [40]

$\mathrm{d} \omega=\mathrm{d} t-\left(\frac{\dot{\phi} \phi^{\prime}}{1-2 G M / r+Q^{2} / r^{2}-\dot{\phi}^{2}}\right) \mathrm{d} r$,

(10) becomes

$$
\begin{aligned}
\mathrm{d} s^{2}= & \left(1-2 G M / r+Q^{2} / r^{2}-\dot{\phi}^{2}\right) \mathrm{d} \omega^{2} \\
& -\left[\frac{\left(\dot{\phi} \phi^{\prime}\right)^{2}}{\left(1-2 G M / r+Q^{2} / r^{2}-\dot{\phi}^{2}\right)}\right. \\
& \left.+\frac{1}{\left(1-2 G M / r+Q^{2} / r^{2}\right)}+\left(\phi^{\prime}\right)^{2}\right] \mathrm{d} r^{2},
\end{aligned}
$$

and (12) will be a black hole metric if $\bar{G}_{00}=\bar{G}_{11}^{-1}$, i.e.

$\dot{\phi}^{2}=\left(\phi^{\prime}\right)^{2}\left(1-2 G M / r+Q^{2} / r^{2}\right)^{2}$.

Let us assume a solution to (13) of the form $\phi(r, t)=\phi_{1}(r)+$ $\phi_{2}(t)$. Then (13) reduces to

$\dot{\phi}_{2}^{2}=\left(\phi_{1}^{\prime}\right)^{2}\left(1-2 G M / r+Q^{2} / r^{2}\right)^{2}=K$. 
$K(\neq 0)$ is a constant $(K \neq 0$ means $k$-essence field will have non-zero kinetic energy). The solution to (14) is

$$
\begin{aligned}
\phi(r, t)= & \phi_{1}(r)+\phi_{2}(t) \\
= & \sqrt{K}\left[r+\frac{\left(2 G^{2} M^{2}-Q^{2}\right) \tan ^{-1} \frac{(r-G M)}{\left.\sqrt{Q^{2}-G^{2} M^{2}}\right)}}{\sqrt{Q^{2}-G^{2} M^{2}}}\right. \\
& \left.+G M \ln \left(Q^{2}-2 G M r+r^{2}\right)\right]+\sqrt{K} t,
\end{aligned}
$$

with $\phi_{1}(r)=\sqrt{K}\left[r+\frac{\left(2 G^{2} M^{2}-Q^{2}\right) \tan ^{-1} \frac{(r-G M)}{\left.\sqrt{Q^{2}-G^{2} M^{2}}\right)}}{\sqrt{Q^{2}-G^{2} M^{2}}}+G M \ln \right.$ $\left.\left(Q^{2}-2 G M r+r^{2}\right)\right]$, and $\phi_{2}(t)=\sqrt{K} t$, and we have taken an arbitrary integration constant to be zero. Therefore the line element (12) becomes

$$
\begin{aligned}
\mathrm{d} s^{2}= & \left(1-\frac{2 G M}{r}+\frac{Q^{2}}{r^{2}}-K\right) \mathrm{d} \omega^{2} \\
& -\frac{1}{\left(1-\frac{2 G M}{r}+\frac{Q^{2}}{r^{2}}-K\right)} \mathrm{d} r^{2},
\end{aligned}
$$

i.e.

$\mathrm{d} s^{2}=\left(\beta-\frac{2 G M}{r}+\frac{Q^{2}}{r^{2}}\right) \mathrm{d} \omega^{2}-\frac{1}{\left(\beta-\frac{2 G M}{r}+\frac{Q^{2}}{r^{2}}\right)} \mathrm{d} r^{2}$,

with $\beta=1-K$. Now going over to the EddingtonFinkelstein coordinates $(v, r)$ or $(u, r)$ along $\theta=0$ i.e., introducing advanced and retarded null coordinates

$v=\omega+r^{*} ; \quad u=\omega-r^{*}$

$r^{*}=\frac{1}{\beta}\left[r+\frac{r_{+}^{2}}{r_{+}-r_{-}} \ln \left|r-r_{+}\right|-\frac{r_{-}^{2}}{r_{+}-r_{-}} \ln \left|r-r_{-}\right|\right]$,

with $r_{+}=\frac{G M}{\beta}+\frac{1}{\beta} \sqrt{(G M)^{2}-\beta Q^{2}}$

and $r_{-}=\frac{G M}{\beta}-\frac{1}{\beta} \sqrt{(G M)^{2}-\beta Q^{2}}$.

Then the line element (17) becomes

$\mathrm{d} s^{2}=\left(\beta-\frac{2 G M}{r}+\frac{Q^{2}}{r^{2}}\right) \mathrm{d} v^{2}-2 \mathrm{~d} v \mathrm{~d} r$

or

$\mathrm{d} s^{2}=\left(\beta-\frac{2 G M}{r}+\frac{Q^{2}}{r^{2}}\right) \mathrm{d} u^{2}+2 \mathrm{~d} u \mathrm{~d} r$

which is analogous to the Robinson-Trautman (RT) metric [41,42] along $\theta=0$ where $\beta$ can take the values $+1,0,-1$. In our case $\beta \neq+1$ because then $K=\dot{\phi}_{2}^{2}=0$ and dark energy is absent. $\beta \neq-1$, i.e. $K \neq 2$ as the total energy density cannot exceed unity $\left(\Omega_{\text {matter }}+\Omega_{\text {radiation }}+\Omega_{\text {darkenergy }}=1\right)$.

Therefore, the only allowed value of $\beta=0$ i.e. $K=1$ and this is a perfectly valid solution because the RT metric allows $\beta=0$. Physically this means that $r_{+}$is pushed to infinity while $r_{-}$is pushed to zero. This implies the radial coordinate $r$ is a time-like coordinate on the whole space-time manifold and the outer horizon a sort of cosmological horizon. Thus, as argued in Ref. [43], the case $K=1$ of (16) does not seem to have a Newtonian limit, which makes it unsuitable for describing astrophysical objects. However, although this may not be suitable as an astrophysical object, it still is a consistent solution of Einstein's equation. In this context, it should be noted that even the Schwarzschild black hole solution is strictly not astrophysically possible because we cannot have static black holes. But still the Schwarzschild solution has been a milestone in understanding various nuances of general relativity. Similar situation prevails also for the Reissner-Nordstrom black hole as charged black holes are highly unlikely in nature for obvious reasons. So any confusion regarding $K$ taking the value +1 should not arise. We shall show below that $K=1$ gives a zero Hawking temperature.

Also note that the solution $\phi(r, t)(15)$ obtained from the black hole conditions $\bar{G}_{00}=\bar{G}_{11}^{-1}$ also satisfies the emergent gravity equation of motion (7) at $r \rightarrow \infty$ along the symmetry axis, $\theta=0: \bar{G}^{00} \partial_{0}^{2} \phi_{2}+\left[\bar{G}^{11}\left(\partial_{1}^{2} \phi_{1}-\Gamma_{11}^{1} \partial_{1} \phi_{1}\right)\right]$ $+\bar{G}^{01} \nabla_{0} \nabla_{1} \phi+\bar{G}^{10} \nabla_{1} \nabla_{0} \phi=0$. The first term vanishes since $\phi_{2}(t)$ is linear in $t$ and the second term within the third brackets vanishes at $r \rightarrow \infty$ because the second term is $-\sqrt{K} Q^{2} \frac{\left(\beta r^{2}-2 G M r+Q^{2}\right)}{r\left(r^{2}-2 G M r+Q^{2}\right)^{2}}$ and the last two terms vanish because $\bar{G}^{01}=\bar{G}^{10}=0$.

So the scalar field that one needs to produce an emergent RN black hole satisfies the equation of motion of emergent gravity (7) only for an infinite coordinate radius along the polar axis. One may question what the geometry discussed has to do with emergent gravity in the first place. The answer is that as the emergent geometry has a scalar field intricately linked with it a priori, having a solution at $r \rightarrow \infty$ is nontrivial in various respects. Let us discuss these.

First note that the solution for the scalar field $\phi,(15)$, does not vanish for $r \rightarrow \infty$ as is usually expected for well behaved fields. Here $\phi_{1}(r \rightarrow \infty)=\sqrt{K}[r+2 G M \ln r+$ $\left.\frac{2 G^{2} M^{2}-Q^{2}}{\sqrt{Q^{2}-G^{2} M^{2}}} \frac{\pi}{2}\right]$

Moreover, if $Q-G M=\alpha$ where $\alpha \rightarrow 0$, so that $\ln r$ is negligible compared to the other terms, then $\phi_{1}(r \rightarrow \infty) \sim$ $\sqrt{K}\left[r+\frac{\sqrt{Q}}{\sqrt{2} \alpha} \frac{\pi}{2}\right]$. All these are solutions of the theory and so deserve mention.

\section{The Hawking temperature for Robinson-Trautman type metric}

We use the tunneling method to calculate the Hawking temperature for (19) [44-50]. A massless particle in a black hole background is described by the Klein-Gordon equation, 
$\hbar^{2}(-\bar{G})^{-1 / 2} \partial_{\mu}\left(\bar{G}^{\mu \nu}(-\bar{G})^{1 / 2} \partial_{\nu} \Psi\right)=0$.

One expands

$\Psi=\exp \left(\frac{i}{\hbar} S+\cdots\right)$

to obtain to leading order in $\hbar$ the Hamilton-Jacobi equation:

$\bar{G}^{\mu \nu} \partial_{\mu} S \partial_{\nu} S=0$.

Assume $\mathrm{S}$ is independent of $\theta$ and $\phi$. Then

$2 \frac{\partial S}{\partial v} \frac{\partial S}{\partial r}+\left(\beta-\frac{2 G M}{r}+\frac{Q^{2}}{r^{2}}\right)\left(\frac{\partial S}{\partial r}\right)^{2}=0$.

The symmetries of the metric permit the action to be written as

$S=-E v+W(r)+J\left(x^{i}\right)$.

Then

$\partial_{v} S=-E ; \partial_{r} S=W^{\prime} ; \partial_{i} S=J_{i} ;$

$J_{i}$ are constants chosen to be zero. Combining (24) and (26)

$-2 E W^{\prime}(r)+\left(\beta-\frac{2 G M}{r}+\frac{Q^{2}}{r^{2}}\right)\left(W^{\prime}(r)\right)^{2}=0$.

Thus

$$
\begin{aligned}
W(r) & =2 \pi i \frac{E}{\beta} \frac{r_{+}^{2}}{r_{+}-r_{-}}+2 \pi i \frac{E}{\beta} \frac{r_{-}^{2}}{r_{-}-r_{+}} \\
& =W\left(r_{+}\right)+W\left(r_{-}\right) .
\end{aligned}
$$

The two values of $W(r)$ correspond to the processes that the particle tunnels through the outer and inner horizons, respectively. Therefore

$S=-E v+2 \pi i \frac{E}{\beta} \frac{r_{+}^{2}}{r_{+}-r_{-}}+2 \pi i \frac{E}{\beta} \frac{r_{-}^{2}}{r_{-}-r_{+}}+J\left(x^{i}\right)$.

The tunneling rates of the outer and inner horizons are

$$
\begin{aligned}
& \Gamma_{+ \text {emergent }}^{\mathrm{RT}} \sim \mathrm{e}^{-2 \operatorname{Im} S+} \sim \mathrm{e}^{-2 \operatorname{Im} W\left(r_{+}\right)} \\
& \quad=\mathrm{e}^{4 \pi \frac{E}{\beta} \frac{r_{+}^{2}}{r_{+}-r_{-}}}=\mathrm{e}^{-\frac{E}{K_{B} T_{+}}}, \\
& \Gamma_{- \text {emergent }}^{\mathrm{RT}} \sim \mathrm{e}^{-2 \operatorname{Im} S-} \sim \mathrm{e}^{-2 \operatorname{Im} W\left(r_{-}\right)} \\
& =\mathrm{e}^{4 \pi \frac{E}{\beta} \frac{r_{-}^{2}}{r_{-}-r_{+}}}=\mathrm{e}^{-\frac{E}{K_{B} T_{-}}} .
\end{aligned}
$$

From these two equations the corresponding Hawking temperatures of the two horizons are, respectively,

$$
T_{+ \text {emergent }}^{\mathrm{RT}}=\frac{\hbar c^{3}(1-K)^{2}}{2 \pi k_{B}} \frac{\sqrt{G^{2} M^{2}-Q^{2}(1-K)}}{\left[G M+\sqrt{G^{2} M^{2}-Q^{2}(1-K)}\right]^{2}}
$$

and

$$
T_{- \text {emergent }}^{\mathrm{RT}}=-\frac{\hbar c^{3}(1-K)^{2}}{2 \pi k_{B}} \frac{\sqrt{G^{2} M^{2}-Q^{2}(1-K)}}{\left[G M-\sqrt{G^{2} M^{2}-Q^{2}(1-K)}\right]^{2}}
$$

Hence, as stated before, the Hawking temperature for this case will vanish as the dark energy density has to be $K=$ $\dot{\phi}_{2}^{2}=1$. So this RT black hole in the presence of dark energy cannot radiate as the dark energy density is constrained to be unity.

\section{Emergent gravity and Kerr metric}

Now take the gravitational metric $g_{\mu \nu}$ to be Kerr. The line element is

$$
\begin{aligned}
\mathrm{d} s_{\text {Kerr }}^{2}= & \left(1-\frac{2 G M r}{\rho^{2}}\right) \mathrm{d} t^{2}+\frac{4 G M r \alpha \sin ^{2} \theta}{\rho^{2}} \mathrm{~d} \phi \mathrm{d} t-\frac{\rho^{2}}{\Delta} \mathrm{d} r^{2} \\
& -\rho^{2} \mathrm{~d} \theta^{2}-\left(r^{2}+\alpha^{2}+\frac{2 G M r \alpha^{2} \sin ^{2} \theta}{\rho^{2}}\right) \sin ^{2} \theta \mathrm{d} \phi^{2},
\end{aligned}
$$

where $\alpha=\frac{J}{G M} ; \rho^{2}=r^{2}+\alpha^{2} \cos ^{2} \theta$ and $\Delta=r^{2}-$ $2 G M r+\alpha^{2}$.

In this context an important point should be stressed. Note that the above metric (34) can be recast (for zero total charge) into the form given in Ref. [51], where the identifications are provided below:

$$
\begin{aligned}
\mathrm{d} s^{2}= & f(r, \theta) \mathrm{d} t^{2}-\frac{\mathrm{d} r^{2}}{g(r, \theta)}+2 H(r, \theta) \mathrm{d} t \mathrm{~d} \phi \\
& -K(r, \theta) \mathrm{d} \phi^{2}-\Sigma(r, \theta) \mathrm{d} \theta^{2},
\end{aligned}
$$

where $f(r, \theta)=\frac{\Delta(r)-\alpha^{2} \sin ^{2} \theta}{\Sigma(r, \theta)}$;

$g(r, \theta)=\frac{\Delta(r)}{\Sigma(r, \theta)} ;$

$H(r, \theta)=\frac{\alpha \sin ^{2} \theta\left(r^{2}+\alpha^{2}-\Delta(r)\right)}{\Sigma(r, \theta)} ;$

$K(r, \theta)=\frac{\left(r^{2}+\alpha^{2}\right)^{2}-\Delta(r) \alpha^{2} \sin ^{2} \theta}{\Sigma(r, \theta)} \sin ^{2} \theta ;$

$\Sigma(r, \theta)=r^{2}+\alpha^{2} \cos ^{2} \theta$;

$\Delta(r)=r^{2}+\alpha^{2}-2 G M r$.

In [51] it has been elaborately shown how the Hawking temperature is independent of $\theta$, although the metric functions depend on $\theta$. In our case the emergent metric $\bar{G}_{\mu \nu}$ contains additional terms but these additional terms are still independent of $\theta$. Therefore, the modified Hawking temperature will still be independent of $\theta$. Therefore we might as well do our evaluation for some fixed $\theta$, i.e. $\theta=0$. We consider the Kerr metric along $\theta=0$. Then (34) becomes [52]

$\mathrm{d} s_{\text {Kerr } ; \theta=0}^{2}=\frac{\Delta}{\rho^{2}} \mathrm{~d} t^{2}-\frac{\rho^{2}}{\Delta} \mathrm{d} r^{2}$, 
where $\rho^{2}=r^{2}+\alpha^{2}$ and $\Delta=r^{2}-2 G M r+\alpha^{2}$. It is to be noted that the same metric (36) was rediscovered in [53-55] using a different route.

As before, we take the $k$-essence field $\phi(r, t)$ to be spherically symmetric in keeping with the usual spherically symmetric Born-Infeld type of lagrangian for the $k$-essence scalar field. This does imply any necessary conflict with the non-spherically symmetric background.

Then one has from (8)

$$
\begin{aligned}
& \bar{G}_{00}=g_{00}-\left(\partial_{0} \phi\right)^{2}=\frac{\Delta}{\rho^{2}}-\dot{\phi}^{2}, \\
& \bar{G}_{11}=g_{11}-\left(\partial_{r} \phi\right)^{2}=-\frac{\rho^{2}}{\Delta}-\left(\phi^{\prime}\right)^{2}, \\
& \bar{G}_{01}=\bar{G}_{10}=-\dot{\phi} \phi^{\prime} .
\end{aligned}
$$

The emergent gravity line element (37) along $\theta=0$ is now $\mathrm{d} s^{2}=\left(\frac{\Delta}{\rho^{2}}-\dot{\phi}^{2}\right) \mathrm{d} t^{2}-\left(\frac{\rho^{2}}{\Delta}+\left(\phi^{\prime}\right)^{2}\right) \mathrm{d} r^{2}-2 \dot{\phi} \phi^{\prime} \mathrm{d} t \mathrm{~d} r$.

Now make a coordinate transformation from $(t, r)$ to $(\omega, r)$ such that

$$
\mathrm{d} \omega=\mathrm{d} t-\left(\frac{\dot{\phi} \phi^{\prime}}{\frac{\Delta}{\rho^{2}}-\dot{\phi}^{2}}\right) \mathrm{d} r .
$$

Then (38) becomes

$$
\mathrm{d} s^{2}=\left(\frac{\Delta}{\rho^{2}}-\dot{\phi}^{2}\right) \mathrm{d} \omega^{2}-\left(\frac{\left(\dot{\phi} \phi^{\prime}\right)^{2}}{\left(\frac{\Delta}{\rho^{2}}-\dot{\phi}^{2}\right)}+\frac{\rho^{2}}{\Delta}+\left(\phi^{\prime}\right)^{2}\right) \mathrm{d} r^{2} .
$$

Equation (40) will a black hole metric if $\bar{G}_{00}=\bar{G}_{11}^{-1}$, i.e.

$$
\dot{\phi}^{2}=\frac{\Delta}{\rho^{2}}+\frac{\Delta^{2}}{\rho^{4}}\left(\phi^{\prime}\right)^{2}-\frac{1}{\rho^{2}} \text {. }
$$

We take a solution of (41) as $\phi(r, t)=\phi_{1}(r)+\phi_{2}(t)$.

Therefore, (41) reduces to

$$
\dot{\phi}_{2}^{2}=\frac{\Delta}{\rho^{2}}+\frac{\Delta^{2}}{\rho^{4}}\left(\phi_{1}^{\prime}\right)^{2}-\frac{1}{\rho^{2}}=K,
$$

where $K(\neq 0)$ is a constant $(K \neq 0$ means that the $k$-essence field will have non-zero kinetic energy). Now from (42) we get $\dot{\phi}_{2}=\sqrt{K}$ and $\phi_{1}^{\prime}=\frac{\left(\sqrt{r^{2}+\alpha^{2}}\right)\left(\sqrt{r^{2}(K-1)+\alpha^{2}(K-1)+2 G M r+1}\right)}{\left(r^{2}-2 G M r+\alpha^{2}\right)}$.

The solution of (42) is

$$
\begin{aligned}
\phi(r, t)= & \phi_{1}(r)+\phi_{2}(t) \\
= & \left(\sqrt{r^{2}(K-1)+\alpha^{2}(K-1)+2 G M r+1}\right) \\
& \times\left(\frac{\sqrt{2 G M r} \tan ^{-1}\left(\frac{r \sqrt{2 G M r}}{\sqrt{\alpha^{2}-2 G M r} \sqrt{\alpha^{2}+r^{2}}}\right)}{\sqrt{\alpha^{2}-2 G M r}}\right) \\
& +\ln \left(2\left(r+\sqrt{\alpha^{2}+r^{2}}\right)\right)+\sqrt{K} t,
\end{aligned}
$$

where $\phi_{1}(r)=\left(\sqrt{r^{2}(K-1)+\alpha^{2}(K-1)+2 G M r+1}\right)$ $\left(\frac{\sqrt{2 G M r} \tan ^{-1}\left(\frac{r \sqrt{2 G M r}}{\sqrt{\alpha^{2}-2 G M r} \sqrt{\alpha^{2}+r^{2}}}\right)}{\sqrt{\alpha^{2}-2 G M r}}+\ln \left(2\left(r+\sqrt{\alpha^{2}+r^{2}}\right)\right)\right)$ and $\phi_{2}(t)=\sqrt{K} t$ and choosing an arbitrary integration constant to be zero. Therefore the line element (40) becomes

$$
\begin{aligned}
\mathrm{d} s^{2} & =\left(\frac{\Delta}{\rho^{2}}-K\right) \mathrm{d} \omega^{2}-\frac{1}{\left(\frac{\Delta}{\rho^{2}}-K\right)} \mathrm{d} r^{2} \\
& =\frac{\beta \Delta^{\prime}}{\rho^{2}} \mathrm{~d} \omega^{2}-\frac{\rho^{2}}{\beta \Delta^{\prime}} \mathrm{d} r^{2},
\end{aligned}
$$

where $\beta=1-K, M^{\prime}=\frac{M}{1-K}, \Delta^{\prime}=\left(r^{2}-2 G M^{\prime} r+\alpha^{2}\right)$ and $\rho^{2}=r^{2}+\alpha^{2}$. Here note that $K \neq 1$ since $\beta$ cannot be zero, as then the metric becomes singular. $K$ cannot be greater than 1 because then the signature of the metric (44) will be wrong. $K \neq 0$ because that would imply that dark energy is absent. Therefore, the only allowed values are $0<K<1$. So there is no question of $K$ approaching 1 and confusion regarding this limit should not arise. It can be shown that for $r \rightarrow \infty$ this metric is an approximate solution of Einstein's equations as the relevant terms fall off as $\frac{1}{r^{3}}$.

We now show that there is a further restriction on the dark energy density $K=\dot{\phi}_{2}^{2}$ if we want the fields $\phi(r, t)$ given by (43) to satisfy the equation of motion (7) along the symmetry axis $\theta=0$ at $r \rightarrow \infty$. For the axi-symmetric case, the equation of motion (7) takes the form $\bar{G}^{00} \partial_{0}^{2} \phi_{2}+$ $\bar{G}^{11} \partial_{1}^{2} \phi_{1}-\bar{G}^{11} \Gamma_{11}^{1} \partial_{1} \phi_{1}+\bar{G}^{01} \nabla_{0} \nabla_{1} \phi+\bar{G}^{10} \nabla_{1} \nabla_{0} \phi=0$. The first term vanishes exactly because $\phi_{2}(t)$ is linear in $t$, and the last two terms vanish because $\bar{G}^{01}=\bar{G}^{10}=0$.

Using the expression for $\Gamma_{11}^{1}=\frac{G M\left(\alpha^{2}-r^{2}\right)}{\left(r^{2}+\alpha^{2}\right)\left(r^{2}-2 G M r+\alpha^{2}\right)}$ the third term for $r \rightarrow \infty$ goes as $\frac{1}{r^{2}}$ and hence may be ignored. The remaining second term for $r \rightarrow \infty$ goes as $\frac{|1-K|^{\frac{3}{2}}}{r}$. As per the Planck collaboration results [56,57], the value of the dark energy density $K$ is about 0.696 . Therefore, the term is negligible as the denominator goes to infinity. Therefore in this limit this term also may be ignored and hence the equations of motion are satisfied. Therefore, $K \neq 0,1$ and $0<K<1$. However $K$ should be very close to unity for equations of motion to be satisfied at large $r$.

\section{The Hawking temperature for Kerr type metric}

Now we go over to the Eddington-Finkelstein coordinates $(v, r)$ or $(u, r)$ along the symmetry axis $\theta=0 . v=\omega+r^{*}$ and $u=\omega-r^{*}, \beta=1-K$ and

$$
r^{*}=\beta^{-1}\left[r+\left(\frac{r_{+}^{2}+\alpha^{2}}{r_{+}-r_{-}}\right) \ln \left|r-r_{+}\right|-\left(\frac{r_{-}^{2}+\alpha^{2}}{r_{+}-r_{-}}\right) \ln \left|r-r_{-}\right|\right],
$$

with $r_{+}=G M^{\prime}+\sqrt{\left(G M^{\prime}\right)^{2}-\alpha^{2}}=\frac{G M}{1-K}+\sqrt{\left(\frac{G M}{1-K}\right)^{2}-\alpha^{2}}$ 
and $r_{-}=G M^{\prime}-\sqrt{\left(G M^{\prime}\right)^{2}-\alpha^{2}}=\frac{G M}{1-K}-\sqrt{\left(\frac{G M}{1-K}\right)^{2}-\alpha^{2}}$. Therefore the line element (44) becomes

$$
\begin{aligned}
\mathrm{d} s^{2} & =\left(\frac{\beta \Delta^{\prime}}{r^{2}+\alpha^{2}}\right) \mathrm{d} v^{2}-2 \mathrm{~d} v \mathrm{~d} r \\
& =\frac{\beta\left(r-r_{+}\right)\left(r-r_{-}\right)}{r^{2}+\alpha^{2}} \mathrm{~d} v^{2}-2 \mathrm{~d} v \mathrm{~d} r .
\end{aligned}
$$

Now proceeding exactly as before we calculate the Hawking temperatures for the two horizons. These are

$$
\begin{aligned}
& T_{+ \text {emergent }}^{K} \\
& \quad=\frac{\hbar c^{3}(1-K)^{2}}{4 \pi k_{B}} \frac{\sqrt{(G M)^{2}-\alpha^{2}(1-K)^{2}}}{(G M)^{2}+G M \sqrt{(G M)^{2}-\alpha^{2}(1-K)^{2}}}
\end{aligned}
$$

and

$$
\begin{aligned}
& T_{- \text {emergent }}^{K} \\
& \quad=-\frac{\hbar c^{3}(1-K)^{2}}{4 \pi k_{B}} \frac{\sqrt{(G M)^{2}-\alpha^{2}(1-K)^{2}}}{(G M)^{2}-G M \sqrt{(G M)^{2}-\alpha^{2}(1-K)^{2}}},
\end{aligned}
$$

where $k_{B}$ is the Boltzmann constant.

\section{Conclusion}

In this work we have determined the Hawking temperatures for emergent gravity metrics having Reissner-Nordstrom and Kerr backgrounds. We have shown that the presence of dark energy modifies the Hawking temperatures. We first do the exercise for the spherically symmetric Reissner-Nordstrom background metric along $\theta=0$. For $\theta=0$ the $k$-essence scalar field satisfies the emergent gravity equations of motion. In this case, the emergent metric can be mapped onto a Robinson-Trautman black hole. When the dark energy density is unity this black hole has zero Hawking temperature, i.e. it does not radiate. We next work with a Kerr background along $\theta=0$ again so that the emergent gravity equations of motion are again satisfied by the dark energy field. The emergent black hole metric satisfies Einstein's equations for large $r$ and always radiates. Our analysis is done in the context of emergent gravity metrics having $k$-essence scalar fields $\phi$ with a Born-Infeld type lagrangian. In both cases the scalar field $\phi(r, t)=\phi_{1}(r)+\phi_{2}(t)$ also satisfies the emergent gravity equations of motion at $r \rightarrow \infty$ for $\theta=0$.

Note that in Ref. [1] the resulting emergent metric had been identified with a topological defect [58-62]. In this work there are no defects in the emergent metrics. There are two event horizons and hence two Hawking temperatures in both cases. But only one of these temperatures, viz., that corresponding to the outer horizon, is observationally relevant.

Open Access This article is distributed under the terms of the Creative Commons Attribution License which permits any use, distribution, and reproduction in any medium, provided the original author(s) and the source are credited.

Funded by $\mathrm{SCOAP}^{3}$ / License Version CC BY 4.0.

\section{References}

1. D. Gangopadhyay, G. Manna, Euro. Phys. Lett. 100, 49001 (2012)

2. S. Hawking, Phys. Rev. Lett. 26, 1344 (1971)

3. L. Smarr, Phys. Rev. Lett. 30, 71 (1973)

4. J. Bardeen, B. Carter, S. Hawking, Commun. Math. Phys. 31, 161 (1973)

5. S. Hawking, Nature (London) 248, 30 (1974)

6. S. Hawking, Commun. Math. Phys. 43, 199 (1975)

7. J. Bekenstein, Phys. Rev. D 7, 2333 (1973)

8. J. Bekenstein, Phys. Rev. D 9, 3292 (1974)

9. G. Gibbons, S. Hawking, Phys. Rev. D 15, 2752 (1977)

10. S.W. Hawking, G.T. Horowitz, S.F. Ross, Phys. Rev. D 51, 4302 (1995)

11. Maulik K. Parikh, Frank Wilczek, Phys. Rev. Lett. 85, 5042 (2000)

12. K. Murata, J. Soda, Phys. Rev. D 74, 044018 (2006)

13. R. Kerner, R.B. Mann, Class. Quant. Grav. 25, 095014 (2008)

14. Z.M. Zheng, Phys. Lett. B 666, 376 (2008)

15. V. Gorini, A. Kamenschik, U. Moschella, Phys. Rev. D 67, 063509 (2003)

16. V. Gorini, A. Kamenschik, U. Moschella, V. Pasquier. arXiv:gr-qc/ 0403062 (2004)

17. L. Rizzi, S. Cacciatori, V. Gorini, A. Kamenschik, O.F. Piatella, Phys. Rev D 82, 027301 (2010)

18. A.Y. Kamenschik, A. Tronconi, G. Venturi, Phys. Lett. B 702, 191 (2011)

19. R.J. Scherrer, Phys. Rev. Lett. 93, 011301 (2004)

20. L.P. Chimento, Phys. Rev. D 69, 123517 (2004)

21. D. Gangopadhyay, S. Mukherjee, Phys. Lett. B 665, 121 (2008)

22. D. Gangopadhyay, Gravit. Cosmol. 16, 231 (2010)

23. D. Gangopadhyay, G. Manna, EPL 100, 49001 (2012)

24. M. Born, L. Infeld, Proc. R. Soc. Lond. A 144, 425 (1934)

25. C. Armendariz-Picon, T. Damour, V. Mukhanov, Phys. Lett. B 458, 209 (1999)

26. C. Armendariz-Picon, V. Mukhanov, P.J. Steinhardt, Phys. Rev. D 63, 103510 (2001)

27. T. Chiba, T. Okabe, M. Yamaguchi, Phys. Rev. D 62, 023511 (2000)

28. C. Armendariz-Picon, E.A. Lim, JCAP 0508, 007 (2005)

29. N. Arkani-Hamed, H.C. Cheng, M.A. Luty, S. Mukohyama, JHEP 05, 074 (2004)

30. N. Arkani-Hamed, P. Creminelli, S. Mukohyama, M. Zaldarriaga, JCAP 0404, 001 (2004)

31. R.R. Caldwell, Phys. Lett. B 545, 23 (2002)

32. J. Callan, G. Curtis, J.M. Maldacena, Nucl. Phys. B 513, 198 (1998)

33. A.D. Rendall, Class. Quant. Grav. 23, 1557 (2006)

34. G.W. Gibbons, Nucl. Phys. B 514, 603 (1998)

35. G.W. Gibbons, Rev. Mex. Fis. 49S1, 19 (2003)

36. M. Visser, C. Barcelo, S. Liberati, Gen. Rel. Grav. 34, 1719 (2002)

37. E. Babichev, V. Mukhanov, A. Vikman, JHEP 09, 061 (2006)

38. E. Babichev, M. Mukhanov, A. Vikman, JHEP 0802, 101 (2008)

39. E. Babichev, M. Mukhanov, A. Vikman, WSPC-Proceedings, February 1, 2008 
40. S. Weinberg, Gravitation and Cosmology, Wiley Student Edition. (John Wiley and Sons (Asia) Pte. Ltd., New York, 2004)

41. H. Stephani et al., Exact Solutions of Einstein's Field Equations, 2nd edn. (Cambridge University Press, Cambridge, 2003)

42. J.B. Griffiths, J. Podolsky, Exat Space-Times in Einstein's General Relativity. (Cambridge University Press, Cambridge, 2009)

43. B.F. Schutz, A First Course in General Relativity, Chap. 8, sect. 4 (Cambridge University Press, Cambridge, 1985)

44. P. Mitra, Phys. Lett. B 648, 240 (2007)

45. B. Chatterjee, A. Ghosh, P. Mitra, Phys. Lett. B 661, 307 (2008)

46. B. Chatterjee, P. Mitra, Phys. Lett. B 675, 640 (2008)

47. P. Mitra, Black Hole Entropy. arXiv:0902.2055

48. E.T. Akhmedov, T. Pilling, A. de Gill, D. Singleton. Phys. Lett. 666, 269 (2008)

49. E.T. Akhmedov, T. Pilling, D. Singleton, Int. J. Mod. Phys. D 17, $2453(2008)$

50. Z. Zhai, W. Liu, Astrophys. Space Sci. 325, 63-67 (2010)

51. R. Kerner, R.B. Mann, Phys. Rev. D 73, 104010 (2006)
52. S. Chandrasekhar, The Mathematical Theory of Black Holes (Oxford University Press, Oxford, 1992)

53. S. Iso, H. Umetsu, F. Wilczek, Phys. Rev. Lett. 96, 151302 (2006)

54. S. Iso, H. Umetsu, F. Wilczek, Phys. Rev. D 74, 044017 (2006)

55. T. Zhu, Gen. Rel. Grav. 44, 1525 (2012)

56. Planck 2013 results. I. Overview of products and scientific results, Planck collaboration. arXiv: 1303.5062

57. Planck 2013 results. XVI. Cosmological parameters, Planck collaboration. arXiv: 1303.5076

58. M. Barriola, A. Vilenkin, Phys. Rev. Lett. 63, 341 (1989)

59. H. Yu, Nucl. Phys. B 430, 427 (1994)

60. D. Gangopadhyay, R. Bhattacharyya, L.P. Singh, Grav. Cosmo. 13, 1 (2007)

61. D. Bazeia, M.A. Gonzalez Leon, L. Losano, J. Mateos Guilarte, Euro. Phys. Lett. 93, 41001 (2011)

62. P. Avelino, D. Bazeia, R. Menezes, J.G.G.S. Ramos, Euro. Phys. J. C 71, 1683 (2011) 